\title{
MicroRNA-494 acts as a tumor suppressor in pancreatic cancer, inhibiting epithelial-mesenchymal transition, migration and invasion by binding to SDC1
}

\author{
YING YANG, XIONG TAO, CHUN-BO LI and CHANG-MIAO WANG \\ Department of General Surgery, The First Affiliated Hospital of Dalian Medical University, \\ Dalian, Liaoning 116011, P.R. China
}

Received February 2, 2018; Accepted May 14, 2018

DOI: $10.3892 / \mathrm{ijo} .2018 .4445$

\begin{abstract}
Pancreatic cancer (PC) is the fourth most common cause of cancer-related mortality in the industrialized world. Emerging evidence indicates that a variety of microRNAs (miRNAs or miRs) are involved in the development of PC. The aim of the present study was to elucidate the mechanisms through which miR-494 affects the epithelial-mesenchymal transition (EMT) and invasion of PC cells by binding to syndecan 1 (SDC1). PC tissues and pancreatitis tissues were collected, and the regulatory effects of miR-494 on SDC1 were validated using bioinformatics analysis and a dual-luciferase report gene assay. The cell line with the highest SDC1 expression was selected for use in the following experiments. The role of miR-494 in EMT was assessed by measuring the expression of SDC1, E-cadherin and vimentin. Cell proliferation was assessed using a cell counting kit (CCK)-8 assay, migration was measured using a scratch test, invasion was assessed with a Transwell assay and apoptosis was detected by flow cytometry. Finally, a xenograft tumor model was constructed in nude mice to observe tumor growth in vivo. We found that SDC1 protein expression was significantly higher in the PC tissues. SDC1 was verified as a target gene of miR-494. The SW1990 cell line was selected for use in further experiments as it had the lowest miR-494 expression and the highest SDC1 expression. Our results also demonstrated that miR-494 overexpression and SDC1 silencing significantly decreased the mRNA and protein expression of SDC1 and vimentin in SW1990 cells, while it increased E-cadherin expression and apoptosis, and inhibited cell growth, migration, invasion and tumor growth. On the whole, the findings of this study demonstrated that miR-494 is able to downregulate SDC1 expression,
\end{abstract}

Correspondence to: Dr Chang-Miao Wang, Department of General Surgery, The First Affiliated Hospital of Dalian Medical University, 222 Zhongshan Road, Dalian, Liaoning 116011, P.R. China

E-mail: wangchangmiaodl@sina.cn

Key words: microRNA-494, syndecan-1, epithelial-mesenchymal transition, pancreatic cancer, migration, invasion thereby inhibiting the progression of PC. These findings reveal a novel mechanism through which miR-494 affects the development of PC and may thus provide a basis for the application of miR-494 in pancreatic oncology.

\section{Introduction}

Pancreatic cancer (PC) is the fourth leading cause of cancer-related mortality worldwide (1) and is associated with a poor prognosis due to liver metastasis (2). PC remains a challenging disease with limited treatment options; at the time of diagnosis, almost $80 \%$ of patients have unresectable tumors with either progressive metastatic growth or locally advanced lesions (3). This phenotypic switch in PC cells may occur due to epithelial-mesenchymal transition (EMT) (4). EMT is a biological process in which cells with a smooth morphology are converted and begin to exhibit mesenchymal characteristics, including the loss of polarity, minimal cell-cell contacts and increased cell projections (5-9). The histological loss of cellular differentiation is a predictive biomarker of poor outcomes in PC (10). Although advances have been made in PC treatment methods, conventional treatments still have undesirable outcomes and patient life expectancy remains poor $(11,12)$. A pattern of differentially expressed microRNAs (miRNAs or miRs) has been developed for gemcitabine-resistant PC cells, and the results have demonstrated that miR-mRNA network-based analysis may provide insight into the molecular mechanisms responsible for drug resistance (13).

miRs are short ( $22 \mathrm{nt}$ in length) non-coding RNAs that regulate gene expression (14). Previous studies have reported that they are involved in the regulation of cancer progression (15-17). By modulating oncogenes or tumor suppressor genes, miRs can affect tumor development (17). A previous study reported that miR-494 was able to downregulate KIT in gastrointestinal stromal tumors (GIST) and that miR-494 overexpression may be a promising treatment strategy for GIST (18). miR-494 has also been shown to suppress colony forming activity and the proliferation of A549 human lung cancer cells (19). The association between miRNAs and syndecan-1 (SDC1) has previously been explored by Parimon et al (20). SDC1 has been implicated in a number of biological functions, and changes in its expression often produce malignant phenotypes arising from elevated 
cell growth, proliferation, invasion and metastasis (21). SDC1 is an important cell surface adhesion biomolecule that is involved in the maintenance of cell morphology, and its dysfunction contributes to cancer progression (22). SDC1 expression is dysregulated in various types of cancer, including head, neck, breast, ovarian and colorectal carcinomas (23-27). The aim of this study was to elucidate the mechanisms through which miR-494 affects EMT and the invasion of PC cells by targeting SDC1.

\section{Materials and methods}

Ethics statement. The present study was approved by the Institutional Review Board of the First Affiliated Hospital of Dalian Medical University in accordance with the Declaration of Helsinki. Written informed consent was obtained from each participant. All experimental protocols were in accordance with the International Convention on the Ethics of Laboratory Animals and relevant national regulations.

Study subjects. A total of 42 patients (25 male, 17 female; aged 31-77 years) who were pathologically diagnosed with PC and underwent exairesis (28) between November, 2013 and November, 2016 at the First Affiliated Hospital of Dalian Medical University (Dalian, China) were included in this study. The inclusion criteria were as follows: i) Patients who were pathologically confirmed to suffer from PC with complete clinical data; ii) patients who had no history of pancreatitis, radiochemotherapy or pancreatic surgery prior to the exairesis; iii) patients who successfully underwent surgery with no complications. The exclusion criteria were as follows: i) Patients who were not pathologically confirmed with PC; ii) patients who had a history of pancreatic disease; iii) patients who exhibited discomfort after surgery. Pancreatitis tissues were collected from 42 patients (22 male, 20 female; aged 30-75 years) with pancreatitis who were treated at the First Affiliated Hospital of Dalian Medical University as the controls. All the tissue samples were obtained by puncture biopsy and R0 (or R1) excision, and no frozen sections were made prior to RNA extraction. However, histopathological examination was performed following surgery to ensure that the tissues obtained were specifically tumor tissues. Fresh specimens were stored in liquid nitrogen, and all cells were cultured in high glucose Dulbecco's modified Eagle's medium (DMEM; HyClone/GE Healthcare Life Sciences, Logan, UT, USA) containing bovine serum albumin (BSA; Invitrogen/Thermo Fisher Scientific, Inc., Waltham, MA, USA) and $1 \%$ streptomycin penicillin (Sigma-Aldrich/Merck KGaA, Darmstadt, Germany) at $37^{\circ} \mathrm{C}$ with $5 \% \mathrm{CO}_{2}$. The medium was changed every 24 to $48 \mathrm{~h}$. The cells were detached with trypsin and passaged. Cells in the logarithmic growth phase were selected for use in further experiments. No patients had received radiochemotherapy prior to surgery and all provided complete clinical data. Following exairesis, the PC tissues and pancreatitis tissues were stored separately in Eppendorf (EP) tubes in liquid nitrogen.

Immunohistochemistry. Tissues were fixed with formaldehyde, dehydrated, embedded in paraffin and cut into serial $4-\mu$ m-thick sections. All sections underwent normal dewaxing and antigen retrieval in citrate buffer under high pressure for
2 min. To block endogenous peroxidase activity, the sections were incubated with $3 \% \mathrm{H}_{2} \mathrm{O}_{2}$ at room temperature for $10 \mathrm{~min}$. The sections were then incubated with the primary monoclonal mouse-anti-human SDC1 antibody (ab128936, 1:8,000; Abcam Inc., Cambridge, MA, USA) overnight at $4^{\circ} \mathrm{C}$, washed 3 times at room temperature and incubated with goat anti-mouse antibody FITC (Johnson Lifescan Inc., Chesterbrook, PA, USA) for $30 \mathrm{~min}$ at $37^{\circ} \mathrm{C}$. Following 3 washes in phosphate buffered saline (PBS), the sections were stained with diaminobenzidine (DAB) (Shanghai Reagent No. 1 Factory, Shanghai, China), redyed and sealed. Known positive sections were used as positive controls and the primary antibody was substituted with PBS as a negative control. Positive SDC1 expression was indicated by brownish-yellow particles in the nucleus or cytoplasm. Scoring was performed using a double blind method (a 13-point scoring method) (29). The amount of SDC1 staining was scored based on the percentage of stained cells and the intensity of staining. The percentage scoring system was as follows: 0 points, no cells stained positive for SDC1; 1 point, $\leq 10 \%$ cells stained positive; 2 points, $11-50 \%$ positive cells; 3 points, $51-80 \%$ positive cells; 4 points, $>80 \%$ positive cells. Staining intensity was scored as follows: 0 point, no staining; 1 point, weak staining; 2 points, moderate staining; 3 points, strong staining. A final score was product of the percentage score and intensity score. To stratify patients for analysis, a score of $>4$ was defined as high/positive SDC1 expression, while $\leq 4$ was low/negative SDC1 expression.

Reverse transcription-quantitative polymerase chain reaction (RT-qPCR). TRIzol reagent (Invitrogen, Carlsbad, CA, USA) was used to extract total RNA from the tissues and also the RNA concentration was determined using TRIzol. Subsequently, $1 \mu \mathrm{g}$ RNA was reverse transcribed using a PrimeScript ${ }^{\mathrm{TM}}$ RT-PCR kit (RR047A; Takara Bio, Inc., Otsu, Japan) to obtain cDNA, which was used as a template for primer design with Primer Premier 5.0 software (amplified fragment size, $156 \mathrm{bp}$ ). Primers were synthesized by Invitrogen. The purity and concentration of the RNA were then detected using a microplate reader (168-1000XC, Model 680; Bio-Rad, Hercules, CA, USA). With $\beta$-actin as an internal reference, the expression levels of SDC1, E-cadherin, Vimentin and miR-494 were determined as previously described (30). The conditions for the reverse transcription were as follows: $37^{\circ} \mathrm{C}$ for $60 \mathrm{~min}$ and $85^{\circ} \mathrm{C}$ for 5 min. A TaqMan PCR assay (Bio-Rad Laboratories, Inc.) was performed, and the thermocycling conditions were as follows: Pre-denaturation at $95^{\circ} \mathrm{C}$ for $3 \mathrm{~min}$, followed by 40 cycles of denaturation at $95^{\circ} \mathrm{C}$ for $10 \mathrm{sec}$, annealing at $60^{\circ} \mathrm{C}$ for $20 \mathrm{sec}$ and extension at $72^{\circ} \mathrm{C}$ for $30 \mathrm{sec}$. The $2^{-\Delta \Delta \mathrm{Cq}}$ method (31) was used to calculate mRNA expression. The primer sequences are presented in Table I.

Cell culture and selection. The non-HPC cell line, HPDE6c7, and the pancreatic ductal adenocarcinoma cell lines (PDACs), namely ASPC-1, SW1990, BXPC-3, CFPAC-1 and PANC-1, were purchased from the Shanghai Cell Bank of the Chinese Academy of Sciences (Shanghai, China) and cultured in complete DMEM containing 10\% BSA, $100 \mathrm{U} / \mathrm{ml}$ penicillin and $100 \mu \mathrm{g} / \mathrm{ml}$ streptomycin at $37^{\circ} \mathrm{C}$ in $5 \% \mathrm{CO}_{2}$ with saturated humidity. The cell line with the highest SDC1 expression was selected for subsequent experimentation. 
Table I. The primer sequences for the RT-qPCR.

\begin{tabular}{ll}
\hline Primer & \multicolumn{1}{c}{ Sequences } \\
\hline miR-494 & F: 5'-TGACCTGAAACATACACGGA-3' \\
& R: 5'-TATCGTTGTACTCCACTCCTTGAC-3' \\
SDC1 & F: 5'-GAGAGGAATCCGGCAGTAGA-3' \\
& R: 5'-GAGCCATCTTGATCTTCAGG-3' \\
E-cadherin & F: 5'-AGACAGGGGTGGAGGAAGTT-3' \\
& R: 5'-GGGCAGGAGTCTAGCAGAAG-3' \\
Vimentin & F: 5'-GACAATGCGTCTCTGGCACGTCTT-3' \\
& R: 5'-TCCTCCGCCTCCTGCAGGTTCTT-3' \\
3-actin & F: 5'-CTCCTTAATGTCACGCAGGATTTC-3' \\
& R: 5'-GTGGGGCGCCCCAGGCACCA-3' \\
\hline
\end{tabular}

RT-qPCR, reverse transcription-quantitative polymerase chain reaction; miR-494, microRNA-494; SDC1, syndecan-1; F, forward; R, reverse.

Dual-luciferase reporter gene. The TIAN amp Genomic DNA kit (Tiangen Biotech Co., Beijing, China) was used for DNA extraction, and the target gene prediction software TargetScan (http://www.targetscan.org/vert_71/) was used to obtain the targets of miR-494. A PCR amplification primer was designed based on the potential site of the 3'-UTR, and the SDC1 3'-UTR segment containing a miR-494 response sequence was constructed. A Dual-Luciferase Reporter Assay System (E1910; Promega, Madison, WI, USA) was used to detect luciferase viability. The site directed mutant sequence without binding site of miR-494 in SDC1 3'-UTR was designed and inserted into the reporter vector. miR-494 mimic was then co-transfected with the SDC1 wild-type sequence (Wt-miR-494/SDC1) or mutant-type sequence (Mut-miR-494/SDC1) into SW1990 cells. At 48 h following transfection, samples were washed twice with PBS and the old medium was discarded. Subsequently, $100 \mu \mathrm{l}$ passive lysis buffer (PLB) were added to each well and shaken at room temperature for $15 \mathrm{~min}$. The cell lysate was collected and analyzed using the system, with program pre-reading time of $2 \mathrm{sec}$, data reading for $10 \mathrm{sec}$, followed by the addition of LARII Stop \&Glo ${ }^{\circledR}$ Reagent (100 $\mu 1$ each time). The prepared LARII Stop \& $\mathrm{Glo}^{\circledR}$ Reagent and luminotron or lighting slab (sample size, $20 \mu \mathrm{l}$ ) was palced in a bioluminescence detector (Glomax 20/20; Promega). The program was run and data were reserved after value readings. The experiment was performed in triplicate with 3 duplicates for each experiment.

Cell culture, grouping and transfection. The PDAC cell line with the highest expression of SDC1 was selected for use in further experiments. The PC cells in the logarithmic growth phase were seeded in a 6 -well plate $\left(1 \times 10^{5}\right.$ cells/well). When cell confluence reached $70 \%$, the culture solution was changed and the cells were washed with serum- and antibiotic-free medium 3 times. A plasmid-liposome mixture (Invitrogen) was added to culture the plates for transfection, shaken for $30 \mathrm{sec}$ and incubated in $5 \% \mathrm{CO}_{2}$ at $37^{\circ} \mathrm{C}$ for $5 \mathrm{~h}$. The medium was replaced with a culture solution containing fresh serum and cultured for $48 \mathrm{~h}$. Lipofectamine 2000 (Invitrogen) was used
Table II. The sequences for the miR-494 mimic, miR-494 inhibitor and $\mathrm{NC}$ plasmid.

Plasmid Sequence

hsa-miR-494 5'-UGAAACAUACACGGGAAACCUC-3' mimic

hsa-miR-494 5'-GAGGUUUCCCGUGUAUGUUUCA-3' inhibitor

Negative control 5'-UUCUCCGAACGUGUCACGUTT-3' sense

Negative control 5'-CAGUACUUUUGUGUAGUACAA-3' antisense

SDC1-siRNA 5'-GACTGCTTTGGACCTAAAT-3'

miR-494, microRNA-494; SDC1, syndecan-1; NC, negative control.

for transfection and the cells were divided into the following groups: Blank (no transfection), miR-494 mimic (transfected with miR-494 mimic), miR-494 inhibitor (transfected with miR-494 inhibitor), negative control (NC, transfected with NC plasmid), SDC1-siRNA (transfected with SDC1-siRNA) and miR-494 inhibitor + SDC1-siRNA (transfected with miR-494 inhibitors and SDC1-siRNA). The miR-494 mimic, miR-494 inhibitor, NC plasmid and SDC1-siRNA were all purchased from the Shanghai GenePharma Co., Ltd. (Shanghai, China). The siRNA sequences are presented in Table II.

Western blot analysis. At $48 \mathrm{~h}$ following transfection, the cells were harvested and lysed with radioimmunoprecipitation assay (RIPA) buffer (Beyotime Institute of Biotechnology, Shanghai, China). Total protein was extracted, and the protein concentration was determined using the BCA method (KeyGene, Wageningen, The Netherlands). The 5X sample loading buffer was mixed with the proteins and boiled for $10 \mathrm{~min}$ in a water bath. Following heating and denaturation, proteins were separated by $7.5 \%$ polyacrylamide gel electrophoresis and transferred onto a polyvinylidene difluoride membrane (PVDF; EMD Millipore, Billerica, MA, USA). The membrane was blocked with 5\% skimmed milk powder for $1 \mathrm{~h}$ followed by incubation with rat anti-human SDC1 (ab34164, 1:8,000; Abcam Inc.), E-cadherin (TA800670, 1:1,000), $\mathrm{N}$-cadherin (TA503775, 1:500) and Vimentin (TA801250, 1:1,000) antibodies (Zhongshan Jinqiao Biotech, Beijing, China) at $4^{\circ} \mathrm{C}$ overnight. The membrane was then washed and incubated with horseradish peroxidase (HRP)-labeled goat anti-mouse secondary antibody (ab97040, 1:5,000; Abcam Inc.) at $37^{\circ} \mathrm{C}$ for $1 \mathrm{~h}$. Enhanced chemiluminescence (ECL) reagents were added to develop the film, and the optical density (OD) of the target bands was analyzed using a gel image processing system (UVP Inc., San Gabriel, CA, USA) with $\beta$-actin as an internal reference.

Cell counting kit (CCK)-8 assay. When cell confluence reached $80 \%$, the cells were washed twice with PBS, detached by $0.25 \%$ trypsin and made into single cell suspension. After cell counting, $3 \times 10^{3}$ to $6 \times 10^{3}$ cells were seeded in each well 
of a 96-well plate (the volume of each well was $100 \mu 1$, with 6 repeated wells in each group). The plate was subsequently incubated for 24,48 or $72 \mathrm{~h}$, following which $10 \mu \mathrm{l} \mathrm{CCK}-8$ reagent (VP757; Dojindo Co., Kumamoto, Japan) were added to each well for a 2-h incubation. An enzyme linked immunosorbent assay reader (Dasit, Milan, Italy) was used to read the OD value of each well at $450 \mathrm{~nm}$. Each experiment was performed in triplicate.

Scratch test. A transferpettor pipette $(200 \mu \mathrm{l})$ was used to make a scratch on the cell surface at $48 \mathrm{~h}$ following cell transfection. Serum-free medium was used to wash the cells 2 times with cell debris removed, following which images were captured under an inverted microscope (time 0 ). The cells were cultured in serum-free medium in a $5 \% \mathrm{CO}_{2}$ incubator with saturated humidity at $37^{\circ} \mathrm{C}$ for $24 \mathrm{~h}$ and the scratch healing was observed and recorded at the same observation site. The healing conditions in each group were compared and the cell migration rate was calculated based on 5 scratch widths.

Transwell assay. A Transwell chamber inserted with a microporous $8 \mu \mathrm{m}$-bore membrane (Costar Corporation, MA, USA) was placed in a 6-well plate. The bottom of the Transwell chamber was coated with $10 \mu \mathrm{g} / \mathrm{ml}$ type I collagen (Sigma-Aldrich Chemical Co., St. Louis, MO, USA) and the chamber was dehydrated under sterile conditions. At $24 \mathrm{~h}$ following transfection, the cells in each group were collected and resuspended at a density of $1 \times 10^{5}$ cells $/ \mathrm{ml}$. Subsequently, $200 \mu \mathrm{l}$ cell suspension was seeded in the apical chamber of the Transwell chamber coated with Matrigel and $800 \mu 1$ DMEM containing $20 \%$ FBS was added to the basolateral chamber, followed by incubation for $24 \mathrm{~h}$. Gel on the upper layer of the microporous membrane was removed using a cotton bud. The cells in the chamber were fixed with paraformaldehyde for $20 \mathrm{~min}$ and stained with crystal violet (Amresco Company, Solon, $\mathrm{OH}, \mathrm{USA}$ ) for $5 \mathrm{~min}$ at $37^{\circ} \mathrm{C}$. Cells were observed, counted under an inverted microscope (magnification, $\mathrm{x} 200$, TE2000; Nikon, Shanghai, China) with representative fields of vision photographed. The number of cells penetrating through the membrane without Matrigel was set as $100 \%$, and the percentage was obtained by comparing the number of cells penetrating through the membrane in each group with the number of cells penetrating through the membrane without Matrigel, which was regarded as the cell invasion ability in vitro. Each group consisted of 3 parallel experiments and each experiment was repeated 3 times.

Flow cytometry. At $48 \mathrm{~h}$ following transfection, the cells were detached using trypsin without ethylene diamine tetraacetic acid (EDTA) and collected in a centrifugation tube. The supernatant was discarded and the cells were washed 3 times with cold PBS and centrifuged $\left(37^{\circ} \mathrm{C}\right.$, $300 \mathrm{x} \mathrm{g}$ ) again. The supernatant was discarded, followed by staining with $1 \mathrm{mg} / \mathrm{ml}$ RNase A and $50 \mu \mathrm{g} / \mathrm{ml}$ of propidium iodide (PI) for $30 \mathrm{~min}$. Flow cytometry (BD Biosciences, San Jose, CA, USA) was performed and the cell cycle was assessed using ModFit software (Bio-Rad Laboratories, Inc.). An Annexin V-fluorescein isothiocyanate (FITC) cell apoptosis kit (M3021; Shanghai Mei Ji Biotechnology Co., Ltd., Shanghai, China) was used to assess apoptosis. In accordance with the manufacturer's instructions, Annexin V-FITC, PI and 4-(2-hydroxyethyl)-1-piperazineethanesulfonic acid (HEPES) buffer solution were mixed at a ratio of 1:2:50 to prepare the Annexin V-FITC/PI dye. A total of $100 \mu \mathrm{l}$ dye was added to per $1 \times 10^{6}$ cells for suspension. The mixture was shaken evenly and incubated at room temperature for $15 \mathrm{~min}$, following which $1 \mathrm{ml}$ HEPES buffer solution was added and the mixture was shaken evenly again. FITC and PI fluorescence were detected using a flow cytometer to assess cell apoptosis. The results were evaluated as follows: Cells in the left upper quadrant were dead cells; cells in the left lower quadrant were negative normal cells; cells in the right quadrant were apoptotic cells; cells in the upper right quadrant were late apoptotic cells and cells in the lower right represented those in early apoptosis.

Xenograft tumors in nude mice. BALB/C female nude mice (8 weeks old, weighing 18-20 g) were purchased from the Laboratory Animal Center, Dalian Medical University and housed under the condition of a constant temperature $\left(25-27^{\circ} \mathrm{C}\right)$ and constant humidity (45-50\%). The SW1990 cells $\left(1 \times 10^{6}\right)$ were resuspended in $200 \mu 1$ normal saline and injected subcutaneously into the back of right hind leg of nude mice with 10 mice for each group. The mice that were injected with the SW1990 cells were assigned into the blank, miR-494 mimic, SDC1-siRNA and miR-494 inhibitor + SDC1-siRNA groups. Tumor formation and growth were observed and recorded regularly. When tumors were clearly visible, the two maximal margins were measured every 5 days using a vernier caliper. Tumor volume was calculated using the formula $\mathrm{V}=\mathrm{L} \times \mathrm{W}^{2} \times 0.5$, where $\mathrm{L}$ is the length and $\mathrm{W}$ is the width of a tumor. When a single tumor reached approximately $1.2 \mathrm{~cm}$ in diameter, the nude mice were sacrificed.

Statistical analysis. SPSS 21.0 software (IBM Corp., Armonk, NY, USA) was used for data analysis. All experiments were repeated 3 times. Data are presented as the means \pm standard deviation. Differences between 2 groups were compared using independent sample t-test. Multiple group comparisons were made using one-way analysis of variance (ANOVA). The least significant difference (LSD) test was employed for pairwise comparisons. The cell growth conditions in each group at different time points were assessed using a repeated measurement analysis of variance. A value of $\mathrm{P}<0.05$ was considered to indicate a statistically significant difference.

\section{Results}

The expression of miR-494 is downregulated and that of SDCl is upregulated in PC tissues. Immunohistochemistry was performed in order to determine the relative expression of SDC1 in PC tissues. Positive SDC1 staining was mainly observed in the nuclei and only partly in cytoplasm (Fig. 1A). The SDC1 positive expression rate in the PC tissues was $85.7 \%(36 / 42)$ compared with $14.4 \%(6 / 42)$ in the pancreatitis tissues $(\mathrm{P}<0.05)$. Subsequently, we validated the inverse association between miR-494 and SDC1 expression by conducting RT-qPCR. The results revealed that miR-494 expression was decreased and SDC1 expression was increased in the PC tissues compared with the pancreatitis tissues $(\mathrm{P}<0.05)$ (Fig. 1B). 
A

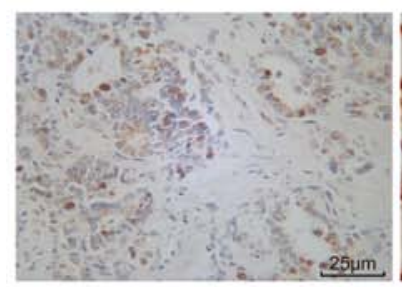

Pancreatitis tissues

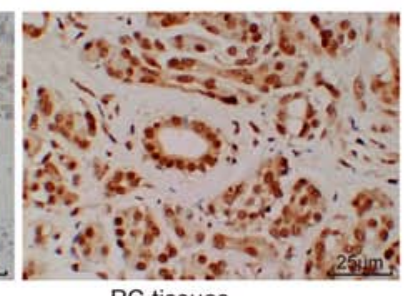

PC tissues
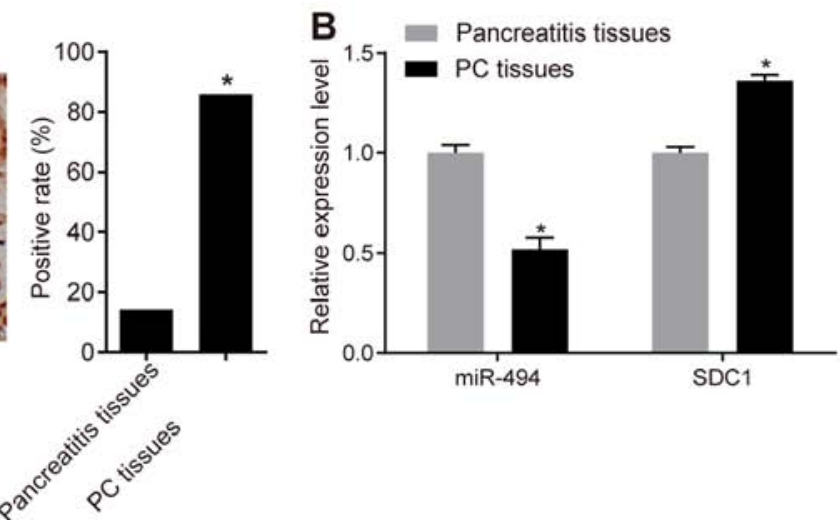

Figure 1. miR-494 is downregulated and SDC1 is upregulated in PC. (A) Immunohistochemistry was used to measure the expression of SDC1 (magnification, $\mathrm{x} 400$ ). (B) RT-qPCR was used to determine the relative expression of miR-494 and SDC1; *P<0.05 vs. the pancreatitis tissues; miR-494, microRNA-494; SDC1, syndecan-1; PC, pancreatic cancer. The data are presented as the means \pm standard deviation, analyzed by independent sample t-test. The experiment was independently repeated 3 times.
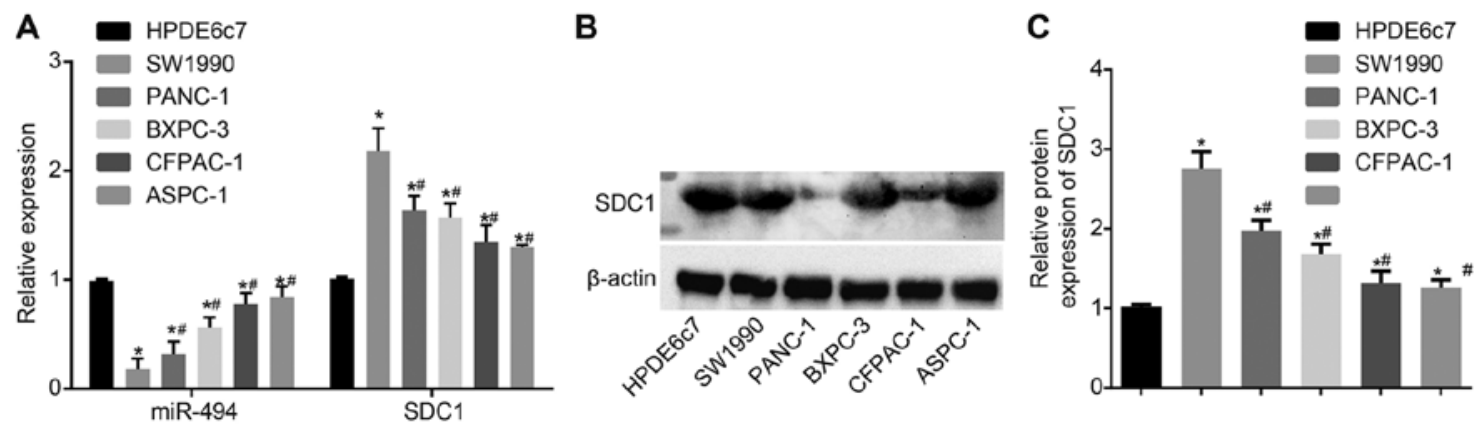

Figure 2. RT-qPCR and western blot analysis demonstrate that SDC1 expression is highest and miR-494 expression is lowest in SW1990 cells. (A) RT-qPCR and (B and C) western blot analysis were used to verify that SDC1 expression is highest and miR-494 expression is lowest in SW1990 cells. "P<0.05 vs. HPDE6c7 cell line; ${ }^{\prime} \mathrm{P}<0.05$ vs. the SW1990 cell line; SDC1, syndecan-1; RT-qPCR: reverse transcription-quantitative polymerase chain reaction. The data are presented as the means \pm standard deviation, analyzed by one-way ANOVA. The experiment was independently repeated 3 times.

A

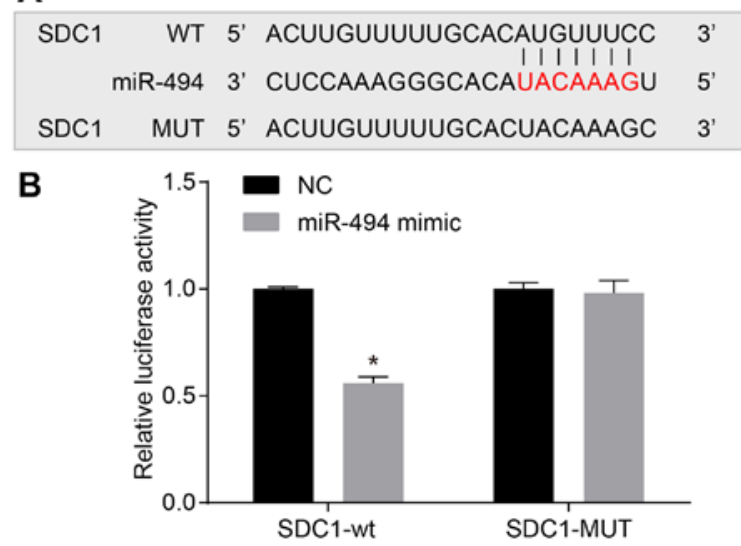

Figure 3. Online target prediction and a luciferase activity assay were used to confirm that SDC12 is the target gene of miR-494. (A) Binding site between SDC12 and miR-494. (B) Relative luciferase activity was decreased following treatment with a combination of miR-494 mimics and SDC1-3'-UTR-WT, suggesting that miR-494 regulates SDC1. ${ }^{*} \mathrm{P}<0.05$ vs. the NC group; NC, negative control; miR-494, microRNA-494; SDC1, syndecan-1. The data are presented as the means \pm standard deviation, analyzed by independent sample t-test. The experiment was independently repeated 3 times.

SW1990 cells have the highest SDC1 expression and lowest miR-494 expression. RT-qPCR was performed to determine the mRNA expression levels of SDC1 in the non-HPC HPDE6c7 cells and PDAC cell lines (ASPC-1, SW1990, BXPC-3, CFPAC-1 and PANC-1). These cell lines exhibited a statistically significant inverse association between SDC1 and miR-494 expression (Fig. 2A). The expression of miR-494 was also assessed. Western blot analysis was used to measure the protein levels expression of SDC1 (Fig. 2B), and the results revealed the same expression pattern as did RT-qPCR. miR-494 expression was lower in the PDAC cell lines than in the HPDE6c7 cells, and the lowest miR-494 expression was observed in the SW1990 cells. The results also revealed that SDC1 expression was upregulated in the PDAC cells compared with the non-PC cells $(\mathrm{P}<0.05)$. Thus, SW1990 was selected for use in further experiments.

SDCl was verified as the target gene for $m i R-494$. Online target prediction was used to determine whether miR-494 is able to directly regulate SDC1, and the results were confirmed by a dual luciferase reporter assay. The results identified a specific binding region between the SDC1 gene and the miR-494 sequence, indicating that SDC1 was the target gene of miR-494 (Fig. 3). To prove that miR-494 affects the target site, WT and MUT sequences lacking the miR-494 combination sites in the SDC1 3'-UTR area were inserted using a reporter plasmid. The miR-494 mimic, WT-miR-494/SDC1 
A

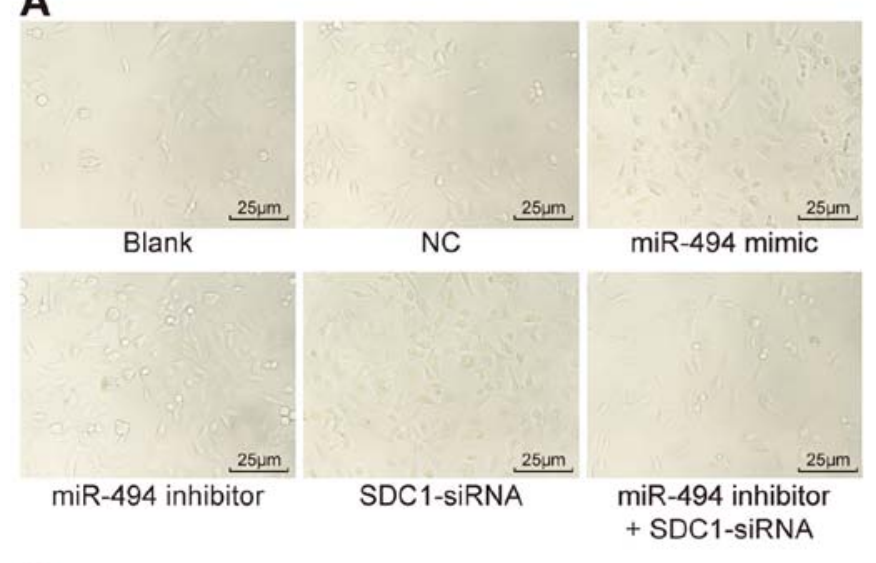

C

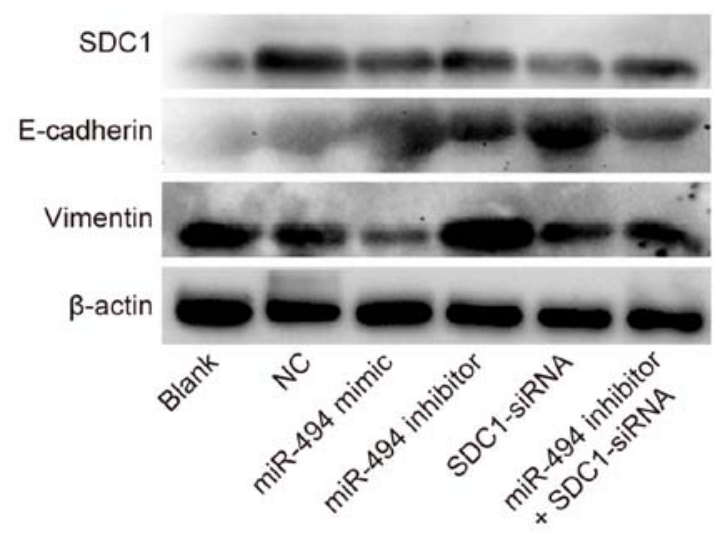

B

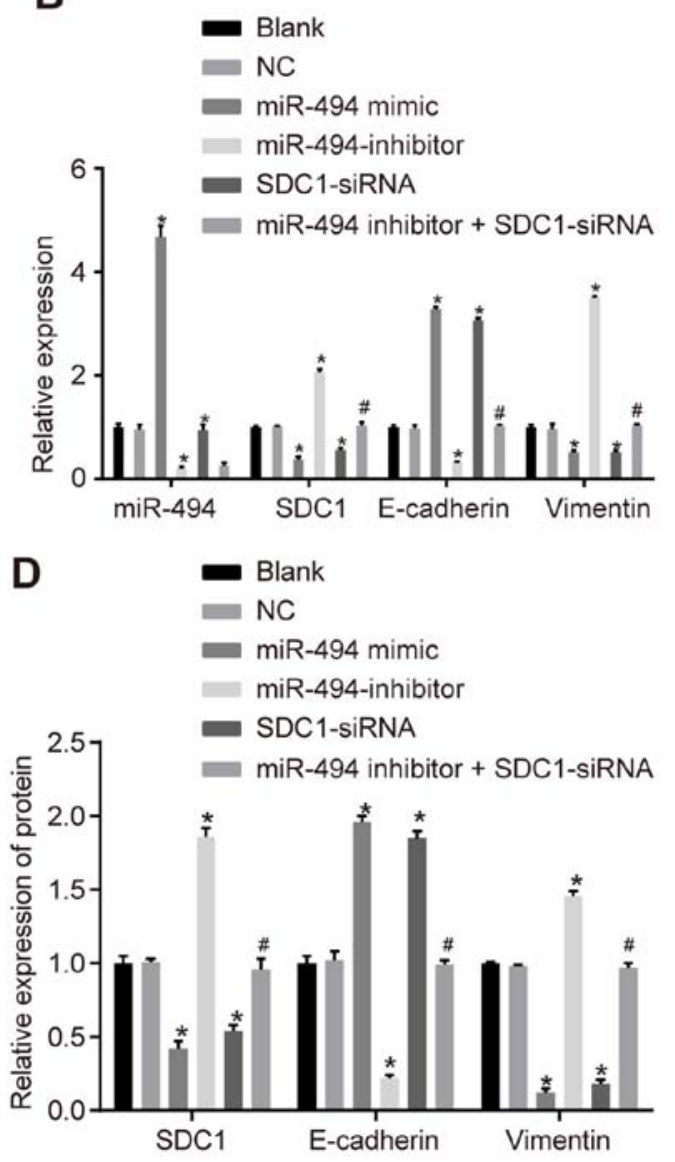

Figure 4. RT-qPCR and western blot analysis were used to demonstrate that miR-494 overexpression inhibits EMT in PC cells. (A) Morphology of SW1990 cells (magnification, x400). (B) RT-qPCR was used to determine the relative expression of miR-494, SDC1, E-cadherin and Vimentin. (C) Grey value of SDC1, E-cadherin and vimentin. (D) Western blot analysis was used to determine the relative expression of miR-494, SDC1, E-cadherin and vimentin. ${ }^{\mathrm{P}<0.05}$ vs. the blank and NC groups; "P<0.05 vs. the miR-494 inhibitor group; NC, negative control; miR-494, microRNA-494; SDC1, syndecan-1; RT-qPCR, reverse transcription quantitative polymerase chain reaction. The data are presented as mean \pm standard deviation, analyzed by one-way ANOVA. The experiment was independently repeated 3 times.

or MUT-miR-494/SDC1 recombined plasmids were co-transfected into the SW1990 cells, and the results revealed that transfection with the miR-494 mimics had no significant effects on luciferase activity in the MUT-miR-494/SDC1 group, whereas the luciferase activity was significantly decreased following transfection in the WT-miR-494/SDC1 group $(\mathrm{P}<0.05)$. These results indicate that miR-494 may directly target SDC1.

Restoration of miR-494 suppressed EMT of SW1990 cells via inhibition of SDC1. Microscopy was used to observe changes in cell morphology at $48 \mathrm{~h}$ following transfection in each group (Fig. 4A). In the NC and blank groups, no obvious interstitial changes were observed. Compared with the NC and blank groups, a loss of cell polarity and widened cell gaps were observed in the miR-494 inhibitor + SDC1-siRNA and the miR-494 inhibitor groups, suggesting that cell adhesion was lost. Some cells in these groups exhibited outstretched pseudopodia or mesenchymal cell-like changes, similar to fibroblasts. Changes in cell morphology were most evident in the miR-494 inhibitor group. No significant interstitial cell transformation was observed in the miR-494 mimic or SDC1-siRNA groups.

RT-qPCR was performed to assess the mRNA expression levels of SDC1, E-cadherin and vimentin in the SW1990 cells, while western blot analysis was employed to measure protein expression (Fig. 4B-D). No significant differences were observed in miR-494, SDC1, E-cadherin or vimentin expression between the blank and NC groups $(\mathrm{P}>0.05)$. Compared with the blank and NC groups, the expression of miR-494 was increased in the miR-494 mimic group, whereas it was decreased in the miR-494 inhibitor and miR-494 inhibitor + SDC1-siRNA groups. Compared with the blank and NC groups, the mRNA and protein levels of SDC1 and vimentin were decreased in the miR-494 mimic and SDC1-siRNA groups, while the expression of E-cadherin was increased $(\mathrm{P}<0.05)$; elevated mRNA and protein levels of SDC1 and vimentin were observed in the miR-494 inhibitor group, while the expression of E-cadherin was decreased $(\mathrm{P}<0.05)$. The mRNA and protein levels of SDC1 and vimentin were lower in the miR-494 inhibitor + SDC1-siRNA group compared with the miR-494 inhibitor group, whereas the expression of E-cadherin was increased $(\mathrm{P}<0.05)$. Taken together, these results suggested that miR-494 overexpression and SDC1 knockdown inhibited EMT in the SW1990 cells, whereas miR-494 knockdown promoted EMT.

miR-494 overexpression suppresses SW1990 cell proliferation by inhibiting SDC1 expression. To better understand the 


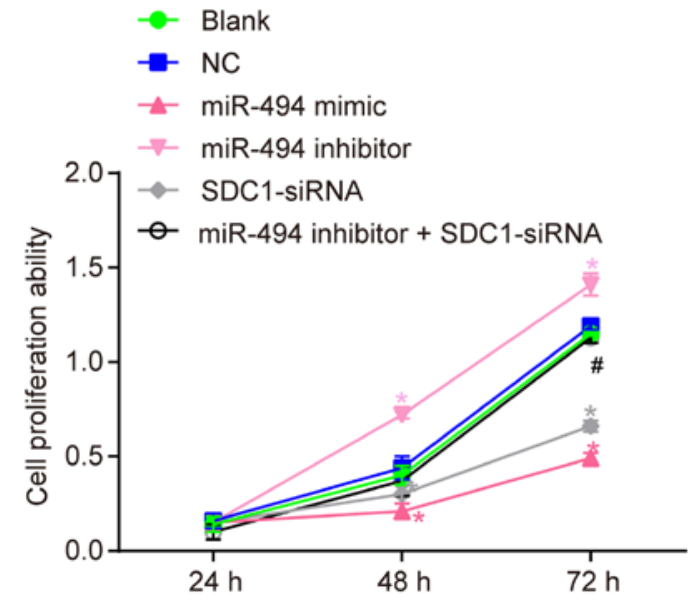

Figure 5. A CCK-8 assay revealed that cell proliferation is suppressed by miR-494 overexpression. ${ }^{*} \mathrm{P}<0.05$ vs. the blank and NC groups; ${ }^{\prime} \mathrm{P}<0.05$ vs. the miR-494 inhibitor group; NC, negative control; miR-494, microRNA-494; SDC1, syndecan-1; CCK8, cell counting kit-8. The data are presented as the means \pm standard deviation, analyzed by one-way ANOVA. The experiment was independently repeated 3 times.

effects of miR-494 on the proliferation of SW1990 cells, a CCK-8 assay was performed. The results (Fig. 5) revealed no significant difference in cell proliferation between the blank and NC groups $(\mathrm{P}>0.05)$. Compared with the blank and NC groups, cell growth was decreased in the miR-494 mimic and SDC1-siRNA groups, as indicated by a lower A450 value at 48 and $72 \mathrm{~h}(\mathrm{P}<0.05)$. Furthermore, the A450 value was increased in the miR-494 inhibitor group $(\mathrm{P}<0.05)$. Compared with the miR-494 inhibitor group, cell proliferation was decreased in the miR-494 inhibitor + SDC1-siRNA group $(\mathrm{P}<0.05)$. These results suggest that miR-494 overexpression or SDC1 silencing suppresses the proliferation of SW1990 cells in vitro. miR-494 silencing enhances cell proliferation, while transfection with SDC1-siRNA reverses this effect.

miR-494 overexpression inhibits SW1990 cell migration and invasion by deregulating SDC1. A scratch test and Transwell assay were conducted to assess the role of miR-494 in the migration and invasion of SW1990 cells. The results (Fig. 6) revealed that, compared with the blank and $\mathrm{NC}$ groups, cell migration and invasion were significantly reduced in the miR-494 mimic and SDC1-siRNA groups, whereas they were elevated in the miR-494 inhibitor group $(\mathrm{P}<0.05)$. The miR-494 inhibitor + SDC1-siRNA group exhibited no significant difference in terms of cell migration and invasion in comparison with the blank and NC groups; however, the miR-494 inhibitor + SDC1-siRNA group exhibited reduced cell migration and invasion compared with the miR-494 inhibitor group. These results suggest that miR-494 overexpression or SDC1 silencing suppresses the migration of SW1990 cells in vitro, while miR-494 silencing enhances cell migration. The effects of miR-494 silencing can be reversed by transfection with SDC1-siRNA.

miR-494 overexpression or SDC1 silencing promotes the apoptosis of SW1990 cells. Flow cytometry was used to detect the cell cycle distribution and apoptosis. The results (Table III and Fig. 7) revealed that there were an increased number of cells in the G0/G1 phase and a decreased number in the $\mathrm{S}$ and G2/M phases in the miR-494 mimic and SDC1-siRNA

A

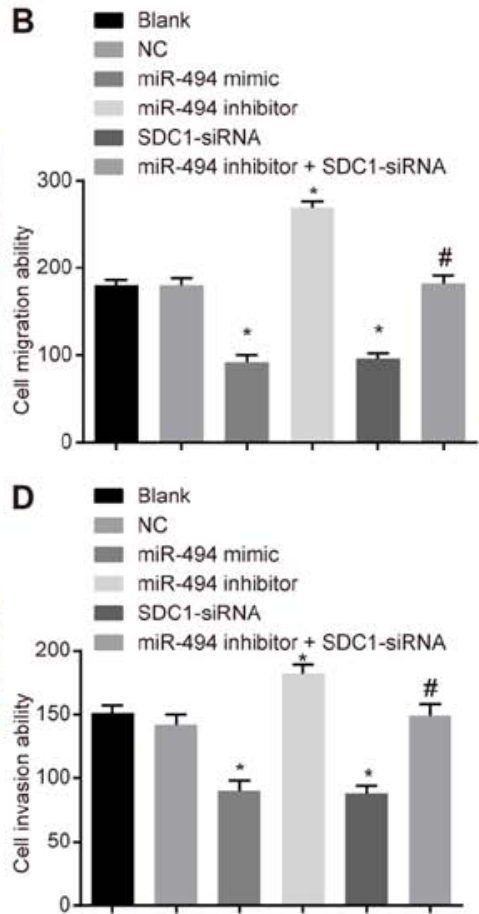

Figure 6. Scratch test and Transwell assays show that cell migration and invasion abilities are inhibited by miR-494 overexpression. (A) A scratch test was used to measure migration. (B) miR-494 upregulation and SDC1 depletion suppress migration. (C) A Transwell assay was utilized to measure the invasion ability. (D) miR-494 upregulation and SDC1 depletion suppress invasion. ${ }^{*} \mathrm{P}<0.05$ vs. the blank and NC groups; ${ }^{\text {P }}<0.05$ vs. the miR-494 inhibitor group; NC, negative control; miR-494, microRNA-494; SDC1, syndecan-1. The data are presented as the means \pm standard deviation, analyzed by one-way ANOVA. The experiment was independently repeated 3 times. 
A
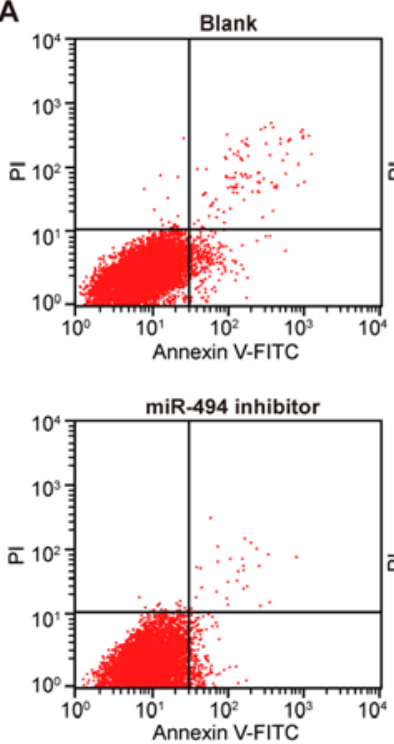
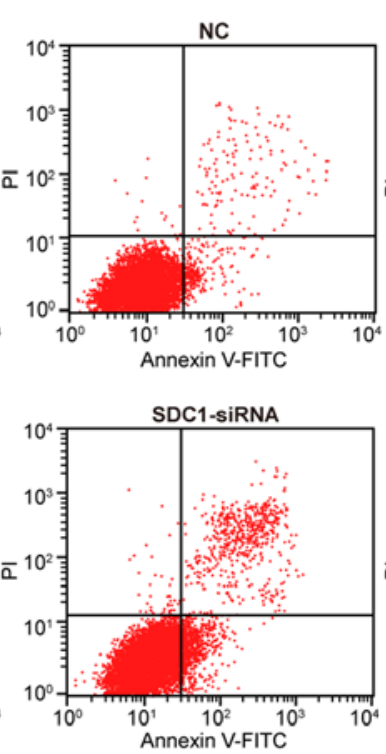

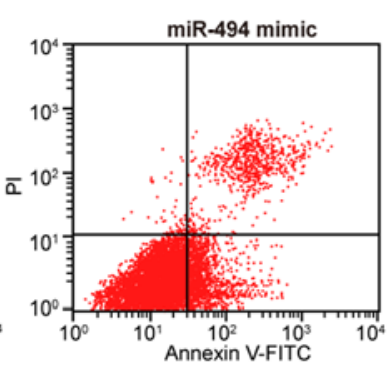

miR-494 inhibitor

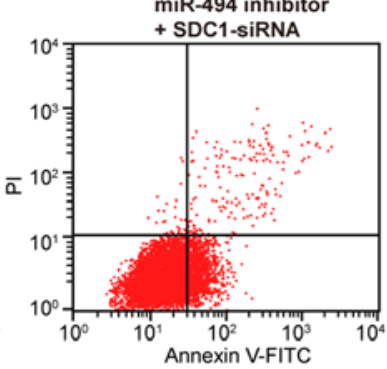

B

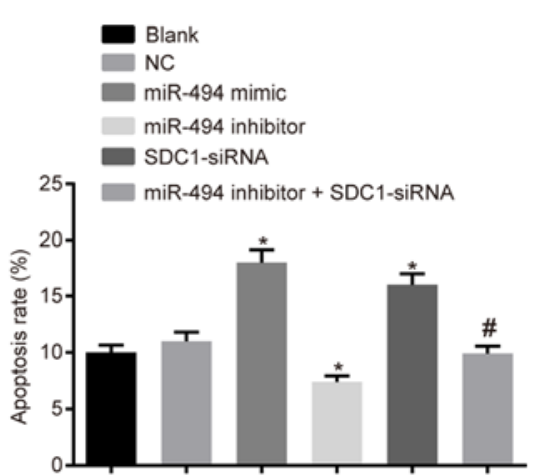

Figure 7. Flow cytometry revealed that miR-494 overexpression promotes cell apoptosis by inhibiting SDC1. (A) Flow cytometry was used to measure apoptosis. (B) miR-494 upregulation and SDC1 depletion promote apoptosis; " $\mathrm{P}<0.05$ vs. the blank and $\mathrm{NC}$ groups; ${ }^{\text {}} \mathrm{P}<0.05$ vs. the miR- 494 inhibitor group; NC, negative control; miR-494, microRNA-494; SDC1, syndecan-1. The data are presented as the means \pm standard deviation, analyzed by one-way ANOVA. The experiment was independently repeated 3 times.

A

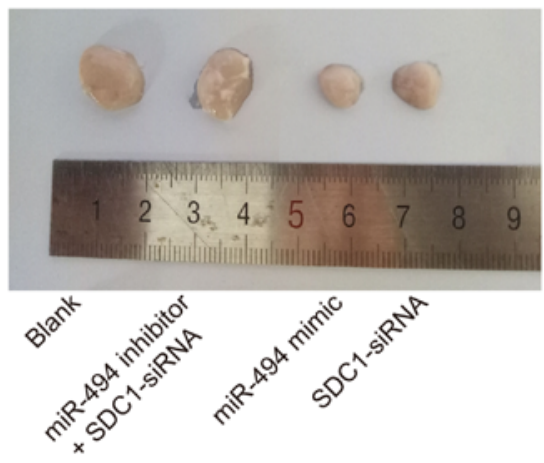

B

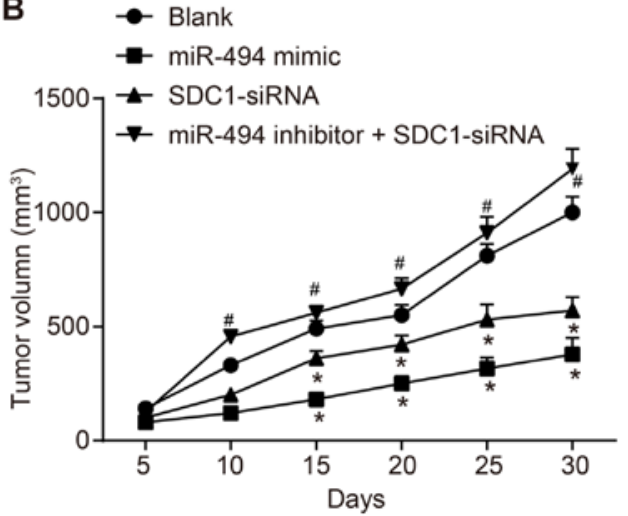

Figure 8. A xenograft tumor model was constructed in nude mice and miR-494 overexpression is found to inhibit tumor growth in vivo. (A) Tumor cells were injected into nude mice following transfection. (B) miR-494 upregulation and SDC1 depletion delay tumor growth; miR-494, microRNA-494; SDC1, syndecan-1. ${ }^{*} \mathrm{P}<0.05$ vs. the blank group; ${ }^{*} \mathrm{P}<0.05$ vs. the SDC1-siRNA group. The data are presented as the means \pm standard deviation, analyzed by one-way ANOVA. The experiment was independently repeated 3 times.

groups compared with the blank and NC groups $(\mathrm{P}<0.05)$. A reduced number of cells was observed in all phases in the miR-494 inhibitor group and apoptosis was also reduced $(\mathrm{P}<0.05)$. Compared with the miR-494 inhibitor group, a significant increase in the number of cells in the G0/G1 phase was observed in the miR-494 inhibitor + SDC1-siRNA group; furthermore, cell apoptosis was increased and the number of cells in $\mathrm{S}$ and $\mathrm{G} 2 / \mathrm{M}$ phase was decreased $(\mathrm{P}<0.05)$. Together, these results suggest that miR-494 overexpression or SDC1 silencing promotes the apoptosis of SW1990 cells; miR-494 silencing exerts an anti-apoptotic effect on the SW1990 cells, and this is reversed by transfection with SDC1-siRNA.

miR-494 overexpression or SDC1 silencing inhibits tumor growth. Finally, in order to verify the effects of miR-494 in PC cells in vivo, we injected transfected PC cells into nude mice to observe tumorigenesis. As shown in Fig. 8, tumor volumes were lower in the miR-494 mimic and the SDC1-siRNA groups compared with the blank group. Tumor volumes in the miR-494 inhibitor + SDC1-siRNA group were significantly increased compared with the SDC1-siRNA group $(\mathrm{P}<0.05)$. In conclusion, miR-494 overexpression or SDC1 silencing inhibits tumor growth.

\section{Discussion}

PC treatment remains a major challenge due to the aggressive progression and high degree of chemoresistance observed in this type of cancer $(3,32)$. Despite advances in treatment methods, the 5-year survival of patients with PC remains $\leq 5 \%$ (33). Thus, the development of novel treatment strategies for PC is of great importance. The finding of this study that miR-494 suppresses the biological behaviors of PC cells suggests that miR-494 may be a potential target for PC treatment. 
Table III. Comparisons of cell number in G0/G1, S and G2/M phases using flow cytometry.

\begin{tabular}{lccc}
\hline Groups & G0/G1 phase & S phase & G2/M phase \\
\hline Blank & $44.06 \pm 1.74$ & $33.24 \pm 3.18$ & $21.69 \pm 1.45$ \\
NC & $44.24 \pm 1.46$ & $35.29 \pm 2.13$ & $20.47 \pm 1.3$ \\
miR-494 mimic & $76.92 \pm 6.19^{\mathrm{a}}$ & $9.68 \pm 5.59^{\mathrm{a}}$ & $13.40 \pm 0.69^{\mathrm{a}}$ \\
SDC1-siRNA & $70.42 \pm 5.45^{\mathrm{a}}$ & $15.46 \pm 4.89^{\mathrm{a}}$ & $14.12 \pm 0.56^{\mathrm{a}}$ \\
miR-494 inhibitor & $31.62 \pm 3.90^{\mathrm{b}}$ & $5.75 \pm 1.21^{\mathrm{b}}$ & $9.46 \pm 1.65^{\mathrm{b}}$ \\
miR-494 inhibitor + SDC1-siRNA & $43.35 \pm 5.65^{\mathrm{b}, \mathrm{c}}$ & $35.38 \pm 5.86^{\mathrm{b}, \mathrm{c}}$ & $21.27 \pm 1.34^{\mathrm{b}, \mathrm{c}}$ \\
\hline
\end{tabular}

${ }^{\mathrm{a}} \mathrm{P}<0.05$, as compared with the blank and $\mathrm{NC}$ groups; ${ }^{\mathrm{b}} \mathrm{P}<0.05$, as compared with the blank and $\mathrm{NC}$ groups; ${ }^{\mathrm{C}} \mathrm{P}<0.05$, as compared with the miR-494 inhibitor group; NC, negative control; miR-494, microRNA-494; SDC1, syndecan-1.

Our results demonstrated that the expression of miR-494 was downregulated and that of SDC1 was upregulated in PC tissues. A recent study reported that miR-494 expression was decreased in gastric carcinoma (GC), indicating that miR-494 can act as an anti-oncogene and plays a role in the pathogenesis of GC (34). Similarly, another study demonstrated that miR-494 was downregulated in oral cancer compared to normal tissues (35). miR-494 expression was found to be associated with tumor progression and has potential as a predictor of a poor prognosis of patients with PC (36). In addition, significantly elevated SDC1 serum levels have been reported in advanced prostate cancer, suggesting that SDC1 shedding is involved in tumor progression (37). The SDC1 expression profile was also found to be significant in colorectal cancer, and may be of use for identifying aggressive colorectal carcinoma (38). Based on these data reported, it can be concluded that patients with PC may exhibit miR-494 downregulation, but an increased level of SDC1.

Vimentin, a major intermediate filament protein, is often overexpressed in epithelial cancers, such as prostate cancer, breast cancer, lung cancer, gastrointestinal tumors and malignant melanomas. Furthermore, vimentin overexpression is typically associated with elevated tumor growth and a poor patient prognosis (39). Vimentin has received attention as a canonical biomarker of EMT (40). Furthermore, its expression is associated with motile prostate cancer cell lines (41), and vimentin overexpression can reduce tumor invasive activity and cell motility via inhibiting PC-3 cells (42). The specific EMT markers of a decreased E-cadherin and an increased vimentin expression are associated with a poor survival $(43,44)$ and invasion $(45)$. Moreover, a previous study reported that transfection with miR-494 mimics upregulated E-cadherin expression and downregulated the expression of $\mathrm{N}$-cadherin and Vimentin in breast cancer cells (46). SDC2 is able to include extracellular E-cadherin shedding, changing the fibroblast-like morphology in colon cancer cell lines (47). Taken together, these reports suggest that miR-494 overexpression or SDC1 silencing decreases vimentin expression, and elevates E-cadherin expression, suggesting that miR-494 can suppress EMT, and the migration and invasion of PC cells.

In this study, following transfection with miR-494 mimics and SDC1-siRNA, the cell growth rate, migration and invasion were decreased, while cell apoptosis was increased. A previous study revealed that a reduced miR-494 expression in PC tissues was associated with tumor progression (36). Furthermore, it has been reported that miR-494 expression is notably decreased in PC tissues, as well as in cell lines, and miR-494 overexpression can significantly suppress PC cell proliferation in vitro and in vivo by inducing apoptosis, senescence and G1-phase arrest (48). miR-494 can inhibit tumorigenesis in breast cancer by targeting PAK1, in gastrointestinal stromal tumors by targeting KIT and in non-small-cell lung cancer by targeting c-Myc (17,49-51). However, the role of miR-494 in cancer cell proliferation may be reversed; by targeting PTEN and BIM, miR-494 contributes to the development of hepatocellular carcinoma and non-small-cell lung cancer, respectively $(51,52)$. Based on these reports, the results of the present study require further validation. In the present study, SDC1 was identified as a target gene of miR-494, while miR-494 was able to negatively target SDC1, suggesting a reciprocal role. Moreover, we hypothesized that miR-494 suppressed EMT, and the migration and invasion of PC cells by inhibiting SDC1. The results of the present study indicate that SDC1 is a functionally linked target gene regulated by miR-494 in PC, suggesting that miR-494 may be used as a novel vertical blockade agent for the treatment of PC. However, there are limitations. miR-494 can also impact PDAC progression via forkhead box protein M1 (FOXM1), NAD-dependent deacetylase sirtuin-1 (SIRT1) and c-Myc $(48,53)$, suggesting that other genes should be considered when evaluating the involvement of miR-494 in PC. As such, further studies are required to validate the results of the present study and gain a broader understanding of the role of miR-494 in PC.

\section{Acknowledgements}

The authors would like to thank all the individuals that provided technical assistance and valuable advice.

\section{Funding}

No funding was received.

\section{Availability of data and materials}

The analyzed data sets generated during the study are available from the corresponding author on reasonable request. 


\section{Authors' contributions}

YY and CMW conceived and designed the study. XT and CMW were involved in data collection. YY and CBL performed the statistical analysis and preparation of figures. YY, XT and CBL drafted the manuscript. All authors have read and approved the final manuscript.

\section{Ethics approval and consent to participate}

The present study was approved by the Institutional Review Board of the First Affiliated Hospital of Dalian Medical University in accordance with the Declaration of Helsinki. Written informed consent was obtained from each participant. All experimental protocols are in accordance with the International Convention on the Ethics of Laboratory Animals and relevant national regulations.

\section{Patient consent for publication}

Not applicable.

\section{Competing interests}

The authors declare that they have no competing interests.

\section{References}

1. Wolfgang CL, Herman JM, Laheru DA, Klein AP, Erdek MA, Fishman EK and Hruban RH: Recent progress in pancreatic cancer. CA Cancer J Clin 63: 318-348, 2013.

2. Ohno K, Nishimori H, Yasoshima T, Kamiguchi K, Hata F, Fukui R, Okuya K, Kimura Y, Denno R, Kon S, et al: Inhibition of osteopontin reduces liver metastasis of human pancreatic cancer xenografts injected into the spleen in a mouse model. Surg Today 40: 347-356, 2010.

3. Kunnimalaiyaan S, Trevino J, Tsai S, Gamblin TC and Kunnimalaiyaan M: Xanthohumol-mediated suppression of Notch1 signaling is associated with antitumor activity in human pancreatic cancer cells. Mol Cancer Ther 14: 1395-1403, 2015.

4. Maupin KA, Sinha A, Eugster E, Miller J, Ross J, Paulino V, Keshamouni VG, Tran N, Berens M, Webb C, et al: Glycogene expression alterations associated with pancreatic cancer epithelial-mesenchymal transition in complementary mode systems. PLoS One 5: e13002, 2010.

5. Hotz B, Arndt M, Dullat S, Bhargava S, Buhr HJ and Hotz HG: Epithelial to mesenchymal transition: Expression of the regulators snail, slug, and twist in pancreatic cancer. Clin Cancer Res 13: 4769-4776, 2007.

6. Sabbah M, Emami S, Redeuilh G, Julien S, Prévost G, Zimber A, Ouelaa R, Bracke M, De Wever O and Gespach C: Molecular signature and therapeutic perspective of the epithelial-to-mesenchymal transitions in epithelial cancers. Drug Resist Updat 11: 123-151, 2008.

7. Yang $\mathbf{J}$ and Weinberg RA: Epithelial-mesenchymal transition: At the crossroads of development and tumor metastasis. Dev Cell 14: 818-829, 2008

8. Buck E, Eyzaguirre A, Barr S, Thompson S, Sennello R, Young D, Iwata KK, Gibson NW, Cagnoni P and Haley JD: Loss of homotypic cell adhesion by epithelial-mesenchymal transition or mutation limits sensitivity to epidermal growth factor receptor inhibition. Mol Cancer Ther 6: 532-541, 2007.

9. Thomson S, Buck E, Petti F, Griffin G, Brown E, Ramnarine N, Iwata KK, Gibson N and Haley JD: Epithelial to mesenchymal transition is a determinant of sensitivity of non-small-cell lung carcinoma cell lines and xenografts to epidermal growth factor receptor inhibition. Cancer Res 65: 9455-9462, 2005.

10. Hruban RH and Adsay NV: Molecular classification of neoplasms of the pancreas. Hum Pathol 40: 612-623, 2009.

11. Vincent A, Herman J, Schulick R, Hruban RH and Goggins M: Pancreatic cancer. Lancet 378: 607-620, 2011.
12. Gutt R, Liauw SL and Weichselbaum RR: The role of radiotherapy in locally advanced pancreatic carcinoma. Nat Rev Gastroenterol Hepatol 7: 437-447, 2010.

13. Shen Y, Pan Y, Xu L, Chen L, Liu L, Chen H, Chen Z and Meng Z: Identifying microRNA-mRNA regulatory network in gemcitabine-resistant cells derived from human pancreatic cancer cells. Tumour Biol 36: 4525-4534, 2015.

14. Zhang XJ, Ye H, Zeng CW, He B, Zhang H and Chen YQ: Dysregulation of miR-15a and miR-214 in human pancreatic cancer. J Hematol Oncol 3: 46, 2010.

15. Esquela-Kerscher A and Slack FJ: Oncomirs - microRNAs with a role in cancer. Nat Rev Cancer 6: 259-269, 2006.

16. Volinia S, Calin GA, Liu CG, Ambs S, Cimmino A, Petrocca F, Visone R, Iorio M, Roldo C, Ferracin M, et al: A microRNA expression signature of human solid tumors defines cancer gene targets. Proc Natl Acad Sci USA 103: 2257-2261, 2006.

17. Budhu A, Ji J and Wang XW: The clinical potential of microRNAs. J Hematol Oncol 3: 37, 2010.

18. Kim WK, Park M, Kim YK, Tae YK, Yang HK, Lee JM and Kim H: MicroRNA-494 downregulates KIT and inhibits gastrointestinal stromal tumor cell proliferation. Clin Cancer Res 17: 7584-7594, 2011

19. Ohdaira H, Sekiguchi M, Miyata K and Yoshida K: MicroRNA-494 suppresses cell proliferation and induces senescence in A549 lung cancer cells. Cell Prolif 45: 32-38, 2012.

20. Parimon T, Brauer R, Schlesinger SY, Xie T, Jiang D, Ge L, Huang Y, Birkland TP, Parks WC, Habiel DM, et al: Syndecan-1 controls lung tumorigenesis by regulating miRNAs packaged in exosomes. Am J Pathol 188: 1094-1103, 2018

21. Gharbaran R: Advances in the molecular functions of syndecan-1 (SDC1/CD138) in the pathogenesis of malignancies. Crit Rev Oncol Hematol 94: 1-17, 2015.

22. Akl MR, Nagpal P, Ayoub NM, Prabhu SA, Gliksman M, Tai B, Hatipoglu A, Goy A and Suh KS: Molecular and clinical profiles of syndecan-1 in solid and hematological cancer for prognosis and precision medicine. Oncotarget 6: 28693-28715, 2015.

23. Hashimoto Y, Skacel M and Adams JC: Association of loss of epithelial syndecan-1 with stage and local metastasis of colorectal adenocarcinomas: An immunohistochemical study of clinically annotated tumors. BMC Cancer 8: 185, 2008.

24. Lundin M, Nordling S, Lundin J, Isola J, Wiksten JP and Haglund C: Epithelial syndecan-1 expression is associated with stage and grade in colorectal cancer. Oncology 68: 306-313, 2005.

25. Lee SH, Choi EJ, Kim MS, Park JW, Lee YS, Kim SY and Kang CS: Prognostic significance of syndecan-1 expression in squamous cell carcinoma of the tonsil. Int J Clin Oncol 19: 247-253, 2014.

26. Lendorf ME, Manon-Jensen T, Kronqvist P, Multhaupt HA and Couchman JR: Syndecan-1 and syndecan- 4 are independent indicators in breast carcinoma. J Histochem Cytochem 59: 615-629, 2011.

27. Kusumoto T, Kodama J, Seki N, Nakamura K, Hongo A and Hiramatsu Y: Clinical significance of syndecan-1 and versican expression in human epithelial ovarian cancer. Oncol Rep 23: 917-925, 2010.

28. Ma Y, Wu Q, Li X, Gu X, Xu J and Yang J: Pancreatic cancer: From bench to bedside. Ann Transl Med 4: 458, 2016.

29. Qian CN, Furge KA, Knol J, Huang D, Chen J, Dykema KJ, Kort EJ, Massie A, Khoo SK, Vanden Beldt K, et al: Activation of the PI3K/AKT pathway induces urothelial carcinoma of the renal pelvis: Identification in human tumors and confirmation in animal models. Cancer Res 69: 8256-8264, 2009.

30. Zhu HN, Zhang SZ, Zhou YK, Wang CL and Wu XB: Cloning and sequence analysis of the coding sequence of $\beta$-actin cDNA from the Chinese alligator and suitable internal reference primers from the $\beta$-actin gene. Genet Mol Res 14: 12159-12167, 2015.

31. Livak KJ and Schmittgen TD: Analysis of relative gene expression data using real-time quantitative PCR and the 2(-Delta Delta C(T)) method. Methods 25: 402-408, 2001.

32. Michl P and Gress TM: Current concepts and novel targets in advanced pancreatic cancer. Gut 62: 317-326, 2013.

33. Saleem M, Kaur S, Kweon MH, Adhami VM, Afaq F and Mukhtar H: Lupeol, a fruit and vegetable based triterpene, induces apoptotic death of human pancreatic adenocarcinoma cells via inhibition of Ras signaling pathway. Carcinogenesis 26: 1956-1964, 2005.

34. He W, Li Y, Chen X, Lu L, Tang B, Wang Z, Pan Y, Cai S, He Y and Ke Z: miR-494 acts as an anti-oncogene in gastric carcinoma by targeting c-myc. J Gastroenterol Hepatol 29: 1427-1434, 2014. 
35. Libório-Kimura TN, Jung HM and Chan EK: miR-494 represses HOXA10 expression and inhibits cell proliferation in oral cancer. Oral Oncol 51: 151-157, 2015.

36. Ma YB, Li GX, Hu JX, Liu X and Shi BM: Correlation of miR-494 expression with tumor progression and patient survival in pancreatic cancer. Genet Mol Res 14: 18153-18159, 2015.

37. Szarvas T, Reis H, Vom Dorp F, Tschirdewahn S, Niedworok C, Nyirady P, Schmid KW, Rübben H and Kovalszky I: Soluble syndecan-1 (SDC1) serum level as an independent pre-operative predictor of cancer-specific survival in prostate cancer. Prostate 76: 977-985, 2016.

38. Kim SY, Choi EJ, Yun JA, Jung ES, Oh ST, Kim JG, Kang WK and Lee SH: Syndecan-1 expression is associated with tumor size and EGFR expression in colorectal carcinoma: A clinicopathological study of 230 cases. Int J Med Sci 12: 92-99, 2015.

39. Satelli A and Li S: Vimentin in cancer and its potential as a molecular target for cancer therapy. Cell Mol Life Sci 68: 3033-3046, 2011.

40. Thiery JP: Epithelial-mesenchymal transitions in tumour progression. Nat Rev Cancer 2: 442-454, 2002.

41. Lang SH, Hyde C, Reid IN, Hitchcock IS, Hart CA, Bryden AA, Villette JM, Stower MJ and Maitland NJ: Enhanced expression of vimentin in motile prostate cell lines and in poorly differentiated and metastatic prostate carcinoma. Prostate 52: 253-263, 2002.

42. Zhao Y, Yan Q, Long X, Chen X and Wang Y: Vimentin affects the mobility and invasiveness of prostate cancer cells. Cell Biochem Funct 26: 571-577, 2008.

43. Javle MM, Gibbs JF, Iwata KK, Pak Y, Rutledge P, Yu J, Black JD, Tan D and Khoury T: Epithelial-mesenchymal transition (EMT) and activated extracellular signal-regulated kinase (p-Erk) in surgically resected pancreatic cancer. Ann Surg Oncol 14: 3527-3533, 2007.

44. Oida Y, Yamazaki H, Tobita K, Mukai M, Ohtani Y, Miyazaki N, Abe Y, Imaizumi T, Makuuchi H, Ueyama Y, et al: Increased S100A4 expression combined with decreased E-cadherin expression predicts a poor outcome of patients with pancreatic cancer. Oncol Rep 16: 457-463, 2006.

45. Nakajima S, Doi R, Toyoda E, Tsuji S, Wada M, Koizumi M, Tulachan SS, Ito D, Kami K, Mori T, et al: N-cadherin expression and epithelial-mesenchymal transition in pancreatic carcinoma. Clin Cancer Res 10: 4125-4133, 2004.
46. Song L, Liu D, Wang B, He J, Zhang S, Dai Z, Ma X and Wang X: miR-494 suppresses the progression of breast cancer in vitro by targeting CXCR4 through the Wnt/ $\beta$-catenin signaling pathway. Oncol Rep 34: 525-531, 2015.

47. Jang B, Jung H, Chung H, Moon BI and Oh ES: Syndecan-2 enhances E-cadherin shedding and fibroblast-like morphological changes by inducing MMP-7 expression in colon cancer cells. Biochem Biophys Res Commun 477: 47-53, 2016.

48. Liu Y, Li X, Zhu S, Zhang JG, Yang M, Qin Q, Deng SC, Wang B, Tian K, Liu L, et al: Ectopic expression of miR-494 inhibited the proliferation, invasion and chemoresistance of pancreatic cancer by regulating SIRT1 and c-Myc. Gene Ther 22: 729-738, 2015.

49. Zhan MN, Yu XT, Tang J, Zhou CX, Wang CL, Yin QQ, Gong XF, He M, He JR, Chen GQ, et al: MicroRNA-494 inhibits breast cancer progression by directly targeting PAK1. Cell Death Dis 8: e2529, 2017.

50. Yun S, Kim WK, Kwon Y, Jang M, Bauer S and Kim H: Survivin is a novel transcription regulator of KIT and is downregulated by miRNA-494 in gastrointestinal stromal tumors. Int J Cancer 142: 2080-2093, 2018.

51. Romano G, Acunzo M, Garofalo M, Di Leva G, Cascione L, Zanca C, Bolon B, Condorelli G and Croce CM: MiR-494 is regulated by ERK1/2 and modulates TRAIL-induced apoptosis in non-small-cell lung cancer through BIM down-regulation. Proc Natl Acad Sci USA 109: 16570-16575, 2012.

52. Liu K, Liu S, Zhang W, Jia B, Tan L, Jin Z and Liu Y: miR-494 promotes cell proliferation, migration and invasion, and increased sorafenib resistance in hepatocellular carcinoma by targeting PTEN. Oncol Rep 34: 1003-1010, 2015.

53. Li L, Li Z, Kong X, Xie D, Jia Z, Jiang W, Cui J, Du Y, Wei D, Huang S, et al: Down-regulation of microRNA-494 via loss of SMAD4 increases FOXM1 and beta-catenin signaling in pancreatic ductal adenocarcinoma cells. Gastroenterology 147: 485-497.e418, 2014 